\title{
L'ÉLÉGANCE LANGAGIÈRE DU HÉRISSON. LE RESPECT DE LA NORME COMME RÉVOLTE DANS L'ÉLÉGANCE DU HÉRISSON DE MURIEL BARBERY ${ }^{1}$
}

\author{
Franciska SKUTTA \\ Université de Debrecen
}

\begin{abstract}
En): The paper, a case study in modern French usage and style, deals with the question of the linguistic norm in Muriel Barbery's novel, The Elegance of the Hedgehog (2006). The point is to show how the self-educated main character, Renée Michel, concierge in an elegant Parisian block of flats, refutes the stereotyped image of her social position through her immense culture and her refined usage of French. The study gives a detailed survey of lexical and grammatical forms of usage in various registers of speech in Renée's linguistic performance and concludes with the idea that Renée's deep respect for the norms of standard French is a special form of revolt against the intellectual negligence characterizing the rich inhabitants always ready to show their superiority over her.
\end{abstract}

Keywords (En): Standard French usage; registers of speech; norm; stylistic deviation.

Mots-clés (Fr): Français standard ; registres linguistiques ; norme ; écart stylistique.

\section{Introduction}

Dans le titre énigmatique du roman de Muriel Barbery, le « hérisson » désigne métaphoriquement le personnage principal, la concierge d'un immeuble parisien de grand standing. Curieusement, ce personnage, madame Renée Michel, veuve quinquagénaire, est une concierge hors norme: en autodidacte, elle a accumulé des connaissances étendues dans les domaines les plus variés - littérature, philosophie, peinture, musique... - mais en même temps, elle dissimule ses qualités intellectuelles pour se protéger contre l'incompréhension, voire les railleries de son entourage. Se montrant stupide et inculte devant les riches habitants de l'immeuble, elle s'enfonce donc dans le petit univers clos de sa loge, où elle vit seule avec son chat nommé Léon (en hommage à Tolstoï) - voilà en effet son côté hérisson. En revanche, cette vie secrète lui permet de garder sa liberté intérieure et de s'abandonner en cachette à ses passions pour les valeurs spirituelles - c'est là incontestablement son élégance. Car pendant que beugle la télévision pour faire écran au monde extérieur, la concierge du 7 rue de Grenelle lit du Marx, étudie la phénoménologie et s'enivre de la musique de Mozart et de Mahler.

Or, dans le roman, au-delà du contenu élevé des expériences intellectuelles et culturelles de Renée, son élégance se manifeste avant tout dans la mise en forme de ses expériences, notamment sur le plan langagier. En effet, son journal ${ }^{2}$ - qui se

\footnotetext{
${ }^{1}$ Paris, Gallimard, 2006. Les numéros de pages renvoient à cette édition.

${ }^{2}$ Le statut du texte de Renée - bien qu'il soit rédigé à la première personne du singulier - n'est pas clair, car ses divisions - par ailleurs munies de titres, chose peu commune pour un journal intime, tout comme l'emploi fréquent du passé simple - ne portent pas les indications habituelles de date ou d'heure, et c'est à peine si l'on y trouve des tournures métanarratives du genre : « Mais je reviens à la nouvelle du jour » (158). Le lecteur doit donc se fier à une remarque assez tardive de Renée ellemême : "Quelle autre raison pourrais-je avoir d'écrire ceci, ce dérisoire journal d'une concierge Echo des études romanes XI/1, $2015 \quad$ - 147 -

ISSN: 1804-8358 (Online)
} 
transforme insensiblement en une série de réflexions purement abstraites pour dire adieu à ses quelques amis au moment où elle se sent mourir à la suite d'un accident - révèle non seulement ses préoccupations intellectuelles, mais aussi ses talents linguistiques, en particulier sa virtuosité dans l'utilisation des différents registres de la langue, impliquant une connaissance profonde de leurs normes.

\section{Norme et attitude}

Certes, la façon dont s'exprime Renée trahit sa manière de penser, et pour en donner un avant-goût, citons deux extraits où une même thématique - celle des portes coulissantes japonaises récemment installées dans un des appartements par son nouveau propriétaire japonais - est commentée par Renée et par son amie Manuela, la bonne portugaise employée dans le même immeuble. Manuela, qui vient de découvrir les avantages de ces portes, fait preuve d'un esprit pragmatique par ses brèves remarques touchant à l'essentiel de leur fonctionnement :

Eh bien, croyez ça, c'est beaucoup plus pratique. Je me demande pourquoi on ne fait pas pareil. On gagne beaucoup de place et c'est moins bruyant. (160)

En revanche, Renée - qui, de manière significative, ne connaît ces portes qu'indirectement, d'après ses expériences esthétiques, à savoir les films du cinéaste japonais Ozu - révèle plutôt un esprit philosophique en réagissant intérieurement aux paroles simples de son amie par une véritable petite dissertation, sous le prétexte des portes coulissantes, sur le rapport de l'homme à l'espace :

Comme c'est vrai. [...] Dès le premier film [d'Ozu], j'avais été fascinée par l'espace de vie japonais et par ces portes coulissantes refusant de pourfendre l'espace et glissant en douceur sur des rails invisibles. Car, lorsque nous ouvrons une porte, nous transformons les lieux de bien mesquine façon. Nous heurtons leur pleine extension et y introduisons une brèche malavisée à force de mauvaises proportions. [...] La porte coulissante, elle, évite les écueils et magnifie l'espace. Sans en modifier l'équilibre, elle en permet la métamorphose. Lorsqu'elle s'ouvre, deux lieux communiquent sans s'offenser. Lorsqu'elle se ferme, elle redonne à chacun son intégrité. Le partage et la réunion se font sans intrusion. La vie y est une calme promenade, lors qu'elle s'apparente chez nous à une longue suite d'effractions. (161-163)

Opposé aux mots simples du langage courant utilisé par Manuela, le vocabulaire de Renée atteint des hauteurs insoupçonnées à propos du même sujet, pourtant quotidien, tant sur le plan de l'abstraction (espace, au lieu de pièces, extension, proportions, équilibre, métamorphose), que sur celui de la poétisation par métaphores (pourfendre l'espace; la porte coulissante, elle, évite les écueils; deux lieux communiquent sans s'offenser; la vie comme une calme promenade ou comme une suite d'effractions). Certains des mots ont en plus une connotation

vieillissante [...] ? » (131). - Par ailleurs, dans le roman, le journal de Renée alterne avec le Journal et les écrits intitulés Pensée profonde de Paloma, adolescente surdouée, solitaire comme Renée, dont elle est l'âme sœur, et qu'elle décrit comme suit: «Mme Michel, elle a l'élégance du hérisson: à l'extérieur, elle est bardée de piquants, une vraie forteresse, mais j'ai l'intuition qu'à l'intérieur, elle est aussi simplement raffinée que les hérissons, qui sont des petites bêtes faussement indolentes, farouchement solitaires et terriblement élégantes » (153). 
spéciale: pourfendre est marqué comme 'vieux' ou 'littéraire' dans les dictionnaires standard, magnifier est 'littéraire', et métamorphose (au lieu de transformation, changement) ne manque pas d'évoquer, dans la mémoire du lecteur, les poèmes d'Ovide.

Un tel usage soigné de la langue dans la bouche d'une concierge produit ici une prose subtile qui permet d'exprimer «l'élévation de l'esprit » (14) à laquelle aspire Renée. Conformément à la thématique «Usage, norme et codification », dans le prolongement des thèses du Cercle linguistique de Prague, je vais donc me concentrer sur le problème de la norme linguistique dans l'extraordinaire performance de Renée ${ }^{3}$, performance qui est à tout moment l'image fidèle de son attitude dans ses rapports aux autres et, d'une manière générale, à la vie, à l'Art et, bien sûr, à la langue elle-même.

Les linguistes pragois ont toujours souligné la nécessité de la «différenciation fonctionnelle» et de l' «enrichissement stylistique» de la langue standard, à l'aide de «moyens d'expression riches et fonctionnellement différenciés, particulièrement dans le lexique et la syntaxe $»^{4}$. Dans l'idiolecte de ce personnage haut en couleur, la richesse du vocabulaire - représentant toute une gamme de registres linguistiques - est particulièrement frappante, mais Renée se montre également «esclave de la grammaire » (84), se lançant dans des commentaires métalinguistiques à propos même d'une erreur de virgule. Vu le nombre fort élevé des usages qui s'écartent, d'une manière ou d'une autre, du langage supposé neutre, employé dans des situations de tous les jours par le locuteur «lambda», pour ainsi dire, je vais d'abord présenter les écarts relevés dans le lexique et dans les constructions grammaticales, passer ensuite aux commentaires faits par Renée «linguiste », pour conclure cette étude par une interprétation possible du sens profond du respect de la norme dans la vie de cette femme hors norme.

\section{Norme et vocabulaire}

«Je suis une fille de la campagne», dit Renée, «dont les espérances de carrière se sont surpassées jusqu'à mener à la conciergerie du 7 rue de Grenelle » (72). Originaire d'un village non identifié, sortant d'un milieu rural modeste ${ }^{5}$, où « on ne causait guère » (41), épousant, à dix-sept ans, un garçon qui travaillait non aux champs, mais déjà à l'usine, Renée n'a pas pris l'habitude de communiquer spontanément dans un patois. En effet, le facteur décisif dans sa formation linguistique, ce n'était ni l'héritage familial, ni un enseignement régulier, mais la lecture passionnée, à l'insu de tous, d'« une part somme toute appréciable de la

\footnotetext{
${ }^{3}$ Il s'agit donc là essentiellement d'une étude de cas et cela concernant un problème précis, de sorte que les autres aspects de cette œuvre très riche, à la fois spirituelle, amusante et émouvante, ne seront pas évoqués dans le présent article.

${ }^{4}$ Cf. notamment les « Thèses générales du Cercle Linguistique de Prague : Principes pour la culture de la langue » (1932), traduites en français et réimprimées dans BÉDARD - MAURAIS (1983: 806). V. également les remarques de B. HAVRÁNEK (1932/1964 : 6-9), sur l'intellectualisation nécessaire de la langue standard.

${ }^{5}$ Renée mentionne effectivement sa «basse extraction » (77). Cf. aussi la dernière image qu'elle revoit avant de mourir : «à l'église du village, les cloches sonnent. Je revois mon père le dos courbé, les bras à l'effort, qui retourne la terre de juin » (351).
} 
littérature mondiale ( 72 ). Ainsi cette provinciale à Paris s'est-elle approprié, contre toute vraisemblance, l'usage naturel du français cultivé, voire recherché et littéraire de notre temps, et avec cela, elle a acquis une conscience linguistique solide, un sûr discernement et une profonde sensibilité concernant les valeurs sémantiques et stylistiques des mots, de même que leur adéquation aux contextes de communication. Certes, son amour de la langue lui fait préférer l'expression soutenue - le journal, d'une écriture réfléchie, en est le cadre idéal pour Renée -, mais en même temps, elle est capable de changer aisément de registre et de justifier au besoin ses choix lexicaux.

\section{Registres « inférieurs » au langage standard cultivé}

Dans ses échanges quotidiens avec les habitants de l'immeuble, Renée, polie mais distante, se borne souvent au strict minimum, non sans une petite satisfaction intérieure de triomphe moral, comme dans cette réponse à une demande un peu sèche : «- Oui, dis-je, en battant des records de concision » (32). Si elle emploie assez rarement, d'ailleurs - des termes populaires ou simplement familiers, c'est plutôt dans ses réflexions solitaires, et avec une intention précise. Ainsi, toujours pour exprimer son mépris envers les riches qui ne connaissent pas la grammaire, elle appelle, par un terme familier, l'une des locataires «la femme d'un grand ponte de l'industrie d'armement $»^{6}(115)$. Mais son style devient particulièrement savoureux lorsqu' elle introduit, de manière inattendue, de telles expressions dans un contexte représentant le langage philosophique, voire technique, de la phénoménologie, dont elle donne une image caricaturale : «Savez-vous que notre conscience ne perçoit pas tout de go mais [...] au moyen de profilages successifs [...] ?» (62 - locution familière), ou encore : «La conscience réflexive influe-telle bénéfiquement sur l'ordre des démangeaisons? Que nenni. » (58), où cet adverbe négatif, perçu aujourd'hui comme 'littéraire' par beaucoup de locuteurs français ${ }^{7}$, est pourtant marqué comme 'vieux' $(T L F)$, 'vieux' et 'dialectal' (Le Robert), employé 'par plaisanterie' $(T L F)$. Dans un autre registre encore, en adressant une plainte comique à son chat, à propos de problèmes philosophiques difficiles à comprendre, Renée utilise un terme d'argot, évocateur de "l'abîme de la phénoménologie » (54) : «Je suis dans la mouise ${ }^{8} »(53)$. Enfin, très rarement, Renée se permet d'employer un terme du langage vulgaire, toujours sur le ton ironique, à propos des riches, chez qui «Manuela a peaufiné au Coton-Tige des chiottes dorées à la feuille qui, en dépit de cela, sont aussi malpropres et puantes que tous les gogues ${ }^{9} \mathrm{du}$ monde $\gg(27)$.

\footnotetext{
${ }^{6}$ Ponte est marqué comme 'populaire' dans le Robert (1966). - Dans les exemples, sauf indication contraire, c'est moi qui souligne.

${ }^{7}$ Cf. aussi le Lexis (1989), avec les qualifications 'classique' et 'littéraire'.

${ }^{8}$ Le mot figure dans le Dictionnaire de l'argot (1990), et reçoit la même qualification dans le TLF (avec 'populaire') et le Robert ; à leur tour, le Petit Robert (1990) et le Lexis indiquent le seul trait 'populaire', tandis que le Robert Micro (1998) qualifie déjà ce mot de 'familier'.

9 Dans le sens de 'lieux d'aisance', le mot gogues (m. pl.) ne figure que dans le Dictionnaire de l'argot. À son tour, le mot chiotte est marqué comme 'trivial' dans le $T L F$, mais plus généralement comme 'populaire' dans le Robert, le Petit Robert et le Lexis, avec, dans ce dernier, cette remarque
} 


\section{Registres du langage soutenu}

Dans le langage de Renée, conformément à ses goûts, les expressions choisies dans les registres soutenus constituent des ensembles bien plus vastes, faisant preuve à la fois de son érudition et de sa sensibilité stylistique. En effet, ses lectures variées lui ont permis d'apprendre, d'une part, la terminologie technique des arts et des sciences, d'autre part, un riche vocabulaire littéraire éventuellement teinté d'archaïsme -, où les choses ordinaires de la vie sont exprimées sur un ton reflétant l'élégance de l'esprit et le respect de la tradition culturelle. Cependant, chacun de ces deux grands domaines lexicaux, amplement représentés dans le journal de Renée, lui offre la possibilité de faire alterner deux types d'usage, à savoir l'emploi d'un terme soit dans son sens premier, dénotatif, soit avec un détournement sémantique (voire formel), souvent dans une intention ironique ou simplement ludique.

\section{Langages techniques}

Quant aux diverses terminologies techniques, on constate encore la prédominance du langage philosophique, dont les termes abstraits - à l'encontre des paroles moqueuses citées plus haut - présentent cette fois certains concepts fondamentaux sur le mode sérieux :

Toute la phénoménologie est assise sur cette certitude : notre conscience réflexive, marque de notre dignité ontologique, est la seule entité en nous qui vaille qu'on l'étudie parce qu'elle nous sauve du déterminisme biologique. (59)

C'est une autre question - d'attitude ou de tour d'esprit - que Renée, avec son penchant à l'ironie, ne peut se retenir ici non plus de couper court à tout ce raisonnement sophistiqué par cette conclusion lapidaire :

Personne ne semble conscient du fait que, puisque nous sommes ${ }^{10}$ des animaux soumis au froid déterminisme des choses physiques, tout ce qui précède est caduc. (59)

Ainsi en est-il de la plupart des termes savants qui, dans les paroles de Renée, ne manquent pas de prendre facilement une double valeur. En les rencontrant dans le texte, le lecteur les reconnaît pour être les noms de concepts scientifiques ou techniques précis, tels incunable (33), dysphonie (14), mécanisme perceptif opérationnel (42), dysfonctionnement rénal (123), filon diamantifère (122) ou quattrocento (214), mais comme ces termes sont souvent placés, intentionnellement, dans des contextes en quelque sorte inadéquats, ils y acquièrent un sens métaphorique ou, du moins, une nuance sémantico-stylistique en plus de la pure dénotation.

Le cas le plus simple, c'est celui où un terme technique est utilisé sans changement de sens, pour rehausser - ironiquement - l'importance d'une situation quotidienne (c'est-à-dire pour mettre en valeur son insignifiance), comme dans

que le mot est proscrit du bon usage. Le mot se trouve aussi dans le Dictionnaire de l'argot. Dans le Robert Micro, il est considéré simplement comme 'familier'.

${ }^{10}$ En italique dans le texte. 
l'exemple suivant où, de plus, un mélange de deux registres, juridique et littéraire, renforce le comique de l'expression: "par l'œil-de-bœuf sis face aux escaliers [...], je m'enquérais discrètement de l'identité du passant» $(17)^{11}$. Dans d'autres cas, les termes techniques servent déjà de métaphores, tels lamentos (90) caractérisant des plaintes sans fin, ou hominiens (101) désignant péjorativement les hommes, selon une vue pessimiste de la société, «champ territorial traversé de grandes ondes hiérarchiques » (101). Renée a d'ailleurs une prédilection pour les termes savants, comme à propos de la «cystite idiopathique interstitielle (124) d'une chatte : «- Mon Dieu mais qu'est-ce ? dis-je, tout alléchée » (124), et d'une manière générale, elle aime méditer sur le langage technique «qui délasse de la littérature », «qui donne l'illusion de la rigueur, le frisson de la simplicité » (123). Cette tendance à l'abstraction et à la précision se manifeste encore par une curieuse habitude de Renée, qui prend la liberté de créer elle-même des expressions pseudo-savantes, un peu maniérées, certes, du type nom abstrait + adjectif épithète dénominal, produisant des effets tantôt esthétisants : « il s'arracha à sa contemplation florale » (91), tantôt franchement comiques : [à propos d'une coiffure] «ma conception capillaire » $(200)^{12}$. Et elle va parfois jusqu'à construire des phrases qui, prises à la lettre, devraient être considérées comme asémantiques : «nous décorons nos intérieurs avec des redondances » (171), où à la place d'un nom désignant un objet concret, on trouve un nom pour le moins surprenant dans le contexte du verbe décorer ${ }^{13}$. Le seul domaine dont les termes techniques échappent à l'emploi ironique, c'est l'Art, où la qualité esthétique du Beau est définie par Renée (à propos des natures mortes des peintres hollandais du XVII ${ }^{\mathrm{e}}$ siècle $^{14}$ ) comme «faisant jaillir le sentiment de l'adéquation » (217) - le contraire, précisément, des inadéquations, des incompatibilités sémantiques que Renée s'amuse à concevoir.

\section{Langage littéraire}

De manière significative, l'adéquation sémantique - voulue - entre les mots et leurs contextes se réalise chez Renée surtout quand elle se sert du langage littéraire pour parler de choses et d'autres. Non qu'elle s'abstienne complètement d'employer un terme dit 'littéraire' sur le ton ironique : «la philosophie s'autorise à ne plus se vautrer que dans le stupre du pur esprit » (61), mais le registre élevé du vocabulaire littéraire lui permet d'embellir l'expression, de la mettre à la hauteur des expériences vécues, qu'il s'agisse d'une sensation agréable:

\footnotetext{
${ }^{11}$ Il arrive que le choix d'un terme juridique s'accompagne nécessairement de l'emploi de la construction impersonnelle, elle-même caractéristique du langage juridique (utilisé ici avec ironie) : «il appert [...] que dominer son Descartes et son Kant n'ouvre pas [...] les portes d'accès à la phénoménologie transcendantale » (52-53).

${ }^{12}$ Il s'agit là d'adjectifs ayant des étymologies savantes, où les étymons latins sont transparents.

${ }^{13}$ Renée emploie cette expression pour résumer l'idée suivante: 'décorer avec plusieurs objets identiques disposés symétriquement'. Cependant, au lieu de l'expression avec des redondances, on associerait normalement au verbe décorer des expressions désignant des objets concrets : "Une nouvelle manière de décorer la table avec des guirlandes de fruits » (Proust, cité dans le TLF, s. v. décorer).

${ }^{14}$ Cf. la simplicité et la précision des termes dans la description d'un tableau de Pieter Claesz : «La gamme chromatique va du jaune à l'ébène. Le fond est d'or mat, un peu sale » (214).
} 
«l'extraordinaire succulence des petites boules dorées [des mirabelles] » (53), du comportement du nouvel habitant japonais de l'immeuble ${ }^{15}:$ «la sereine aménité avec laquelle M. Ozu se tient devant moi » (188) ou des rapports humains représentés dans un film du cinéaste Ozu : «le père, donc, qui va bientôt mourir, devise avec sa fille » (103), une scène qui, mêlée à d'autres souvenirs de Renée, «embrase [s]on cœur quiet» (111). Deviser et quiet, quiétude, connotés positivement, sont par ailleurs des mots récurrents chez Renée, qui s'en sert toujours à propos des quelques personnes qui lui sont chères, comme Manuela : « Nous rions et devisons [...] dans la quiétude des vieilles amitiés» (30). De même, c'est avec des termes littéraires respectueux que Renée évoque le souvenir de son mari, un homme sans culture littéraire, mais qu'elle aimait pour sa droiture et sa bonté : «mon défunt mari » (16), «Lucien trépassé » (17) expriment bien la tendresse qu'elle lui a vouée pendant leur vie commune et après.

La fréquentation des textes littéraires a certainement contribué à nuancer la sensibilité linguistique de Renée, qui ne se contente pas de recourir au vocabulaire recherché existant et de commenter les phrases des autres, tels ses passages favoris de Guerre et Paix; au contraire, elle s'engage avec le plus grand naturel dans des jeux linguistiques, voire littéraires, depuis l'invention de formes nouvelles jusqu'aux intertextualités les plus subtiles. Ses motifs peuvent varier ici aussi entre l'ironie mordante et l'enthousiasme sincère.

L'invention de formes nouvelles - déjà à l'œuvre dans les expressions pseudosavantes, mais dont les constituants sont des mots français réels - peut se faire dans le domaine de la dérivation, sur le modèle de formes dérivées existantes, mais donnant des mots non attestés dans les dictionnaires standard, tels autodidactie (51 - cf. 'ineptie', 'inertie') ${ }^{16}$ ou «Léon [le chat mal nourri] est désormais le reproche muet félinifié » (185 - cf. 'fortifié', 'lénifié'). Un autre type d'invention, c'est la déformation de dictons et de locutions bien connus : à partir de 'noblesse oblige', Renée crée une expression ironique méchante pour ridiculiser un défaut d'articulation chez une habitante peu sympathique : "Cancrelat buccal oblige, [elle] ne dit pas : les Chinois mais les Chunois. J'ai toujours rêvé de visiter la Chune. C'est [...] plus intéressant que de se rendre en Chine» (155); ou ailleurs, par un simple changement de préposition, elle transforme la locution 'du coq à l'âne' en une expression d'auto-ironie gentille : "Et la conversation [avec M. Ozu] se poursuit ainsi [...] avec l'enthousiasme du coq et de l'âne» (246). En revanche, le processus inverse - forme existante, sens nouveau - se produit lorsque Renée attribue à un mot un sens figuré non attesté dans les dictionnaires, mais évident d'après le contexte : « un géronte toasté aux UV ( 84 - 'bronzé') ou [un matin, cet homme] «a l'air tout débronzé » $(82 \text { - 'découragé' })^{17}$.

Mais l'invention linguistique ne s'arrête pas là chez Renée, qui prend plaisir à employer diverses figures de rhétorique pour nommer et décrire une personne ou un objet. Ainsi, par métonymie, le docteur attristé avec ostentation, et qui oublie de

\footnotetext{
${ }^{15}$ Le seul des habitants, avec Paloma, qui reconnaisse, respecte et apprécie l'intelligence de Renée.

${ }^{16}$ Seul le Lexis indique une forme semblable : autodidaxie.

${ }^{17}$ Toaster au sens de 'faire griller du pain' apparaît dans le $T L F$; ce même dictionnaire et le Lexis (mais non les dictionnaires Robert) indiquent débronzé, -e avec le sens de 'qui a perdu son bronzage', marqué comme 'familier' dans le TLF.
} 
se moucher, devient Nez qui Coule (83), et la dame qui ne connaît pas les règles de la ponctuation s'appellera madame pourriez-vous-virgule-réceptionner (143), tandis que des métaphores frappantes apparaissent dans les formulations suivantes : «ce rosaire laïc qu'on appelle télécommande» (103) ou, à propos du caractère de $\mathrm{M}$. Ozu, « la collusion de l'appétit, de la lucidité et de la magnanimité figure un inédit et savoureux cocktail» (248). De telles expressions, à connotations positives ou négatives, montrent en même temps un certain penchant à l'exagération, qui peut se manifester par des tours emphatiques, comme ces hyperboles: «le pragmatisme de Manuela a quelque chose de galactique » (195) ; "Manuela [...] vouait à la jeune Mme Meurisse une haine racinienne» (75) ; «au prix d'un effort herculéen, je parviens à dire quelque chose» (147) ; « une colossale tranche de pain croulant sous le beurre et la confiture de mirabelles ne parvient qu'à aiguiser mon dantesque appétit» (343). Les trois derniers exemples contenant des références culturelles explicites témoignent en même temps d'une inclination de Renée à intégrer ses connaissances littéraires ou autres dans sa propre production langagière. Certes, tout son journal respire son amour et sa vénération pour les arts, et la littérature en particulier, mais il y a une différence entre les méditations « théoriques » de Renée, et l'emploi spontané d'un terme (en général, la forme adjectivale d'un nom propre bien connu), évocateur de l'atmosphère d'un univers fictif familier aux gens cultivés. Les références semblables peuvent devenir plus complexes encore lorsque plusieurs personnages d'œuvres littéraires ou musicales sont nommés dans une même phrase par laquelle Renée décrit, non sans une exagération ironique, sa propre performance linguistique (une réponse étonnée à une remarque de $\mathrm{M}$. Ozu) : «-Non? dis-je en renouvelant avec brio l'exécution du 'laisser mourir sur les lèvres' dont je partage l'art avec Phèdre, Bérénice et cette pauvre Didon » (186). Le personnage de Didon, dans l'opéra de Purcell, revient plus loin, dans un contexte d'exaltation musicale quand, après la citation en anglais des paroles de son air magnifique, « la mort de Didon ${ }^{18}$, Renée analyse en phonéticienne et musicienne admirative, «l'enchaînement incroyablement dense des sons », atteignant à « une beauté née de la subversion de l'articulation phonétique et de la transgression du soin que le langage verbal met d'ordinaire à distinguer les sons» $(301)^{19}$. Mais l'intertextualité la plus subtile apparaît là où il n'y a que suggestion, des bribes d'allusions, par certains assemblages de mots, à des œuvres cachées derrière les paroles de Renée. En effet, décrivant le rituel du thé qu'elle prend avec Manuela dans le calme de sa loge - moment exceptionnel de félicité dans leur vie de «subalternes par définition anonymes » (83) - Renée a des élans poétiques qui

\footnotetext{
${ }^{18}$ Tout en citant le texte anglais avec admiration (p. 300), Renée est capable d'en jouer : dans un rare moment de bonheur, elle change «forget my fate » en « envy my fate» (341).

${ }^{19}$ On peut relever d'autres exemples cités en langues étrangères, soit encore en rapport avec la musique : "Confutatis maledictis, Flammis acribus addictis!», du Requiem de Mozart (239 - en italique dans le texte), ou sur le mode plaisant: «face au danger, fight, flee ou freeze. Je freeze. J'aurais bien flee, mais [...]» (238 - en italique dans le texte).
} 
l'emportent vers le Macbeth de Shakespeare ${ }^{20}$ et un roman de Faulkner, par les mots bruit ('sound') et fureur ('fury') :

Au-dehors, le monde rugit ou s'endort, les guerres s'embrasent, les hommes vivent et meurent, des nations périssent, d'autres surgissent qui seront bientôt englouties et, dans tout ce bruit et toute cette fureur, dans ces éruptions et ces ressacs, tandis que le monde va, s'enflamme, se déchire et renaît, s'agite la vie humaine. (93),

tandis que la tasse de thé donne la paix, une paix dont la sensation est peinte ici par une image empruntée à Baudelaire :

Le silence se fait, on entend le vent qui souffle au-dehors, les feuilles d'automne bruissent et s'envolent, le chat dort dans une chaude lumière. Et, dans chaque gorgée, se sublime le temps. (94)

\section{Norme et grammaire}

«Je suis esclave de la grammaire, me dis-je, j'aurais dû appeler mon chat Grévisse. » (84) - cette phrase pourrait être la devise de Renée, qui se montre une excellente élève de l'auteur du Bon usage ${ }^{21}$. Comme dans le choix des mots, elle distingue clairement les registres linguistiques dans l'utilisation des formes et des constructions grammaticales, et là encore sa préférence va aux solutions du langage soigné, voire littéraire ${ }^{22}$, qu'il s'agisse des temps et des modes verbaux, ou de l'ordre des termes dans la proposition. En effet, chaque fois où elle relate des événements passés, elle fait de la littérature en employant le passé simple, temps verbal qui apparaît même avec une valeur spécifique de 'passé éloigné' dans cette phrase sentencieuse: "primates nous fûmes, primates nous restons » (112). L'hypothèse - au lieu d'une subordonnée introduite par (même) si - est exprimée à plusieurs reprises par d'autres moyens, plus littéraires, tels le subjonctif imparfait ou le présent de l'indicatif en inversion : «Eussé-je le loisir de [...]» (57); «Vous surprend-on, vous agace-t-on, vous déconcerte-t-on, qu'il vous faut conserver le même raffinement dans l'impassibilité » (337). En dehors de ce type de construction, l'inversion, marque évidente du langage soigné, apparaît ici maintes fois en proposition incise, mais, curieusement, donnant une impression d'hypercorrection parfois comique, car à côté de formes en elles-mêmes recherchées, telles argué-je (186), réponds-je (29, 240), poursuis-je (338), on y trouve également des verbes qui n'appartiennent pas aux verbes déclaratifs attendus : «- Merci, parviens-je à murmurer» (341), ou «- Je... kof... kof..., toussé-je» (337). À part l'inversion, toujours dans le domaine de l'ordre des termes, on peut relever encore une construction typique, notamment l'antéposition

\footnotetext{
20 "Life [...] is a tale / Told by an idiot, full of sound and fury, / Signifying nothing" (paroles de Macbeth, Acte V, Scène V).

${ }^{21}$ Par contraste, et en se moquant de son lamentable bredouillement dans un moment d'émotion, Renée dit : «- Je..., dis-je à M. Ozu [...], je... Enfin... Vous savez, le Requiem ? J'aurais dû appeler mon chat Padsyntax » (240).

${ }^{22}$ Cette tendance générale n'exclut pas pour autant l'emploi de constructions grammaticales relâchées, familières, par exemple l'omission du pronom personnel sujet conjoint et une proposition incise sans inversion : «Devriez lire l'Idéologie allemande, je lui dis » (13).
} 
de l'adjectif épithète pour une emphase affective, extrêmement fréquente ici, qu'il s'agisse de caractériser une personne : « un pathétique snob» (32), «sa fantasque cadette» (104), un objet: «la répugnante et embryonnaire moustache » (14), «nos risibles chemins » (122), des émotions ou des comportements : « une fruste vanité » (101), «la patiente obstination » (216), «la morne frénésie du temps » (189), mais surtout des entités abstraites : «le fragile équilibre» (112), «sa désarmante évidence» (53), «la harassante nécessité » (187), «la cosmique conscience de l'absurdité » (187), et enfin la littérature elle-même : «quelle plus noble distraction, n'est-ce pas, quelle plus distrayante compagnie, quelle plus délicieuse transe que celle de la littérature ? (129).

\section{Commentaires métalinguistiques}

Cette phrase élogieuse sur le rôle de la littérature dans la vie de l'homme peut servir de transition vers les «méditations linguistiques» de Renée, et ses jugements relatifs à la norme à observer dans les différentes situations de communication. En effet, son sens de la norme et des variations, aiguisé par sa relation intime avec cette « distrayante compagnie » qu'est la littérature, provoque souvent chez elle des réactions affectives intenses qu'elle traduit en paroles par des explications circonstanciées.

Certaines observations de Renée se rapportent à des phénomènes linguistiques qui, considérés en eux-mêmes, donnent un plaisir esthétique à qui sait les apprécier. À propos du style de Tolstoï, elle admire la «fluidité de la phrase » (110) ; un diagnostic formulé dans la terminologie médicale est pour elle «une phrase récréative, qui sonne bien à l'oreille » (123), et par moments, elle aime lire les notices de médicaments pour le répit que donnent ces textes d'où «sont absents l'effort vers le beau, la souffrance créatrice et l'aspiration sans fin et sans espoir à des horizons sublimes » (123).

Dans d'autres cas, le phénomène linguistique qui retient l'attention de Renée est commenté à la lumière des rapports interpersonnels, notamment du point de vue de l'expression linguistique de la politesse. Il lui arrive notamment de se lancer dans de véritables «exercices de style ( sans référence à Queneau), cherchant la phrase adéquate à une situation, et justifiant en même temps le choix ou le rejet d'une solution par des considérations sur les registres linguistiques et sur le ton des expressions. Ainsi, avant d'aller aux toilettes lors du dîner amical chez M. Ozu, elle se pose la question «Comment demande-t-on ceci dans le monde ?» (231), puis elle passe en revue mentalement les désignations possibles, autant de variations sur un thème : l'endroit, les toilettes, les cabinets, les gogues (qui ne lui paraît pas idoine), faire pipi (ne se dit pas à table), pour enfin choisir commodités (231-232) ${ }^{23}$.

Mais ce qui enchante Renée et la fait réfléchir, c'est, d'une part, un certain archaïsme des mots et des constructions grammaticales dans les formules de politesse :

\footnotetext{
${ }^{23}$ Même exercice pour tenter de refuser poliment un cadeau de M. Ozu (184-185) et son invitation à dîner (187-188).
} 
Chabrot [le médecin] s'exprime d'une façon dont je raffole - pouvez-vous éconduire les fâcheux ? - et cela me trouble. Cette désuétude polie me plaît. [...] Ce type m'indispose mais sa langue est délectable (84),

d'autre part, une certaine liberté individuelle qui révèle la personnalité du locuteur:

- Vous n'êtes pas occupée, nous prendrions le thé maintenant ?

Cette désinvolture dans la concordance des temps, cet usage du conditionnel à la forme interrogative sans inversion du verbe, cette liberté que Manuela prend avec la syntaxe parce qu'elle n'est qu'une pauvre Portugaise contrainte à la langue de l'exil, ont le même parfum de désuétude que les formules contrôlées de Chabrot (90).

Cependant, cette liberté langagière ne doit pas devenir blessante, comme cela se produit dans l'échange où l'un des riches habitants s'adresse à sa concierge de manière peu courtoise :

- Pourriez-vous me l'apporter immédiatement [...] ?

- Oui, dis-je, en battant des records de concision, encouragée en cela par la sienne et par l'absence de s'il vous plaît que la forme interrogative et conditionnelle ne saurait, d'après moi, excuser totalement.

- C'est très fragile, ajoute-t-il, faites attention, je vous prie.

La conjugaison de l'impératif et du «je vous prie » n'a pas non plus l'heur de me plaire, d'autant qu'il me croit incapable de telles subtilités syntaxiques et ne les emploie que par goût, sans avoir la courtoisie de supposer que je pourrais m'en sentir insultée (32).

Ces paroles de Pierre Arthens provoquent déjà (sans qu'il s'en doute) une critique acerbe de la mentalité des riches :

C'est toucher le fond de la mare sociale que d'entendre dans la voix d'un riche qu'il ne s'adresse qu'à lui-même et que, bien que les mots qu'il prononce vous soient techniquement destinés, il n'imagine même pas que vous puissiez les comprendre (32-33).

Seulement, le mépris de cet homme (et des autres habitants) ne peut porter atteinte à la dignité de Renée, qui conclut cette réflexion en ces termes: «Dieu saura lequel de nous deux s'humilie le plus » (33). Et chaque fois où une faute grammaticale dans la bouche d'un riche la choque, elle fait une double critique, celle de l'usage fautif et celle du locuteur négligent. Le ton n'est pas nécessairement amer, ni violent, au contraire, il peut être distant et ironique, comme à propos de l'omission du pronom sujet :

- Pourquoi les ordures sentent jusque dans la cour?

Que Bernard Grelier et l'héritière d'une vieille famille de la Banque puissent se soucier des mêmes choses triviales et ignorer conjointement l'utilisation du pronom personnel postverbe que la forme interrogative requiert jette sur l'humanité un éclairage nouveau (72),

où la tension née entre deux grandeurs incommensurables, le pronom et l'humanité, crée une prodigieuse hyperbole ironique dans cette «langue délectable » de Renée. 
Mais la critique peut se faire sur le mode sérieux (sauf la métaphore peu flatteuse pour désigner la dame concernée), comme lorsque Renée donne une explication sociologique pour justifier sa position :

- Le paillasson [...] n'a pas été nettoyé. Pouvez-vous pallier $\grave{a}^{24}$ ça ? me demande la poule [Mme Rosen, au « cancrelat buccal »].

[...] Certes, il m'arrive à moi aussi d'user de la faute, bien que ce soit comme d'une arme.

- C'est $u n^{25}$ espèce d'infarctus ? avais-je demandé à Chabrot pour faire diversion de mes manières saugrenues.

Je ne suis donc pas si sensible qu'un écart mineur me fasse perdre raison. Il faut concéder aux autres ce que l'on s'autorise à soi-même; en outre, Jacinthe Rosen et sa blatte dans la bouche sont nées à Bondy dans une barre d'immeubles aux cages d'escalier pas propres et $\mathrm{j}$ 'ai partant pour elle des indulgences que je n'ai pas pour madame pourriez-vous-virguleréceptionner (142-143).

Tandis que Renée est prête à montrer une tolérance linguistique envers ceux qui viennent de milieux sociologiques plus ou moins défavorisés, elle est absolument intransigeante avec les personnes - les riches - qui auraient pu se permettre de s'instruire pour s'approprier les règles de l'usage correct, de sorte que sa critique sévère est sans appel à propos d'une faute d'orthographe - un «blasphème » (116) - dans un petit mot écrit au dos d'une carte de visite :

\footnotetext{
Madame Michel, pourriez-vous, réceptionner les paquets du pressing cet après-midi $?^{26}$ $[\ldots]$

Je ne m'attendais pas à une telle sournoiserie dans l'attaque [...] les bavouilleries ${ }^{27}$ sur vélin de Sabine Pallières transperçant la phrase d'une virgule devenue poignard. [...] Sabine Pallières eût-elle été une bonne portugaise [...], une concierge frâ̂chement émigrée de Puteaux ou bien une déficiente mentale [...] que j'aurais pu pardonner de bon cœur cette nonchalance coupable. Mais Sabine Pallières est une riche.[...] Les faveurs du sort ont un prix. Pour qui bénéficie des indulgences de la vie, l'obligation de rigueur dans la considération de la beauté n'est pas négociable (114-115).
}

\section{Conclusion}

Bien que ces commentaires de Renée parlent pour eux-mêmes, on peut tenter de donner une interprétation de son rapport très personnel à la langue et à la norme.

《 Je suis à mon archétype une trahison permanente » (28) - la justesse de cette constatation de Renée se manifeste entre autres dans ses extraordinaires compétences et performances linguistiques, ces dernières étant toujours guidées par sa conscience aiguë de la norme dans le langage cultivé. Au fond, c'est ce qui lui permet de varier avec aisance les différents registres, tout comme les tons sérieux et ludiques, les usages normés et par moments subversifs de la langue. En même temps, en observatrice perspicace, Renée enregistre les performances linguistiques des gens autour d'elle, qu'elle commente ensuite dans un esprit souvent prescriptif. On retrouve ici - mutatis mutandis - le principe de deux

\footnotetext{
${ }^{24}$ En italique dans le texte.

${ }^{25}$ En italique dans le texte.

${ }^{26}$ En italique dans le texte.

${ }^{27}$ Bavouillerie est un mot créé par Renée.
} 
aspects complémentaires de la norme, celui de 'modèle consensuel à suivre' et celui d' 'objet de réflexion et de codification'.

Or, c'est surtout dans les réflexions de Renée sur la norme et les comportements linguistiques des locuteurs que se révèlent ses rapports à la langue, et conjointement à la société. Car pour elle, la langue - objet d'amour et de vénération - a une double dimension, esthétique et sociologique : sa beauté, sans être immuable dans le temps, doit être sauvegardée dans ses usages variés, devoir sacré qui fait appel à la responsabilité de la société, de ceux en particulier à qui la vie a épargné les misères de la pauvreté. Cette pensée, Renée l'expose dans un élan à la fois poétique et politique, en adressant un véritable hymne à la langue :

La langue, cette richesse de l'homme, et ses usages, cette élaboration de la communauté sociale, sont des œuvres sacrées. Qu'elles évoluent avec le temps, se transforment, s'oublient et renaissent tandis que, parfois, leur transgression devient la source d'une plus grande fécondité, ne change rien au fait que pour prendre avec elles ce droit du jeu et du changement, il faut au préalable leur avoir déclaré pleine sujétion ${ }^{28}$. Les élus de la société, ceux que la destinée excepte de ces servitudes qui sont le lot de l'homme pauvre, ont partant cette double mission d'adorer et de respecter la splendeur de la langue. [...] Aux riches, le devoir du Beau. Sinon, ils méritent de mourir (115-116).

Cette position radicale de Renée - on s'en doute bien - n'amène aucun acte violent, car cette femme intelligente, qui connaît sa place dans la société et n'a aucune illusion sur un possible changement de ses conditions, trouve un autre moyen pour résister à la vulgarité ambiante : c'est le culte, intérieur et solitaire, de la beauté de la langue. Tandis que les autres se révoltent en général contre les contraintes de la norme, par ignorance, négligence, paresse intellectuelle et manque d'élévation, Renée se révolte contre ces attitudes indignes à l'aide $d u$ respect de la norme, seul garant de cette beauté émouvante que nous offrent la saveur des mots et l'élégance de la grammaire. Posséder cette richesse immatérielle est un trésor pour Renée, qui résume ainsi ses aspirations : «j’ai voué ma vie à la quête de l'intemporel » (228).

\section{BIBLIOGRAPHIE}

BARBERY Muriel (2006), L'élégance du hérisson, Paris, Gallimard.

BÉDARD Édith, MAURAIS Jacques (éds.) (1983), La norme linguistique, Québec, Conseil de la Langue Française. L'article cité est la version française d'une traduction tchèque-anglais faite par Paul L. Garvin; traductrices: Andrée Thouin et Francine Paradis.

HAVRÁNEK Bohuslav (1932/1964), The Functional Differentiation of the Standard Language, in : GARVIN Paul L. (éd.), A Prague School Reader on Esthetics, Literary Structure, and Style, Washington, Georgetown University Press, p. 316 (traduction de Paul L. Garvin).

\footnotetext{
${ }^{28}$ Les idées de Renée concernant l'évolution inévitable de la langue, et partant de la norme - sousentendue ici dans l'idée de 'transgression' -, font écho à cette thèse pragoise : « La codification de la norme grammaticale, si elle est bien comprise, doit tenir compte du fait que même la langue standard évolue inévitablement; les efforts en vue de codifier la langue ne doivent pas viser à bloquer cette évolution [...] » (BÉDARD - MAURAIS, 1983 : 804).
} 